\title{
Ciclo de Mejora en la asignatura Estadística Avanzada. Inferencia Estadística: Estimación puntual de los parámetros de una distribución de Probabilidad
}

\author{
LUIS ANDRÉS ZAMBRANA \\ Departamento de Economía Aplicada II \\ Universidad de Sevilla \\ lazambrana@us.es \\ ORCID: https://orcid.org/0000-0003-3606-7816 \\ D.0.1.: http://dx.doi.org/10.12795/JDU.2018.i01.89 \\ Pp.: 1588-1605
}

\section{Resumen}

En esta comunicación describiré la aplicación de un Ciclo de Mejora Docente (CMD) en la asignatura de Estadística Avanzada y basado en el planteamiento de un caso real al que los estudiantes han aplicado las herramientas de Inferencia Estadística. Para ello han recorrido el proceso que debería seguir cualquier investigador que, partiendo del interés en conocer una realidad cuya información no se encuentre registrada en publicaciones o estadísticas oficiales, ha de diseñar la forma de recoger información, poner en práctica el procedimiento de recogida y aplicar las herramientas de las que nos dota la inferencia estadística para, finalmente, llegar a conclusiones numéricas sobre la realidad objeto de interés. 
Palabras claves: Estadística Avanzada; Aprendizaje en Disciplinas Técnicas; Grados en Administración y Dirección de Empresas, Investigación y Técnicas de Mercado y Economía.

\section{Descripción del contexto de la intervención}

El CMD lo apliqué en la asignatura Estadística Avanzada, de segundo curso del Grado en Administración y Dirección de Empresas, llevándolo a cabo en los dos grupos que imparto (de los ocho existentes). Los estudiantes ya cursaron, en primer curso, la asignatura de Estadística Descriptiva, por tanto, están familiarizados con muchos de los conceptos que forman parte de nuestros contenidos. La asignatura es cuatrimestral y tiene seis créditos, los grupos objeto del ciclo son de mañana (grupos 1 y 3 ) y las clases tienen lugar los martes y viernes en bloques de dos horas.

En el grupo1 hay 76 alumnos matriculados de los que 33 son repetidores, mientras que en el 3 el número de matriculados es de 77, siendo 30 repetidores. A clase suelen asistir alrededor de 40 alumnos. Las aulas de ambos grupos son iguales, con bancas corridas de seis plazas, ancladas al suelo, y dispuestas en tres columnas de 9. El piso del aula es plano y frente a la bancada, alzada sobre la cota cero del aula, se encuentra el encerado para el profesor con mesa y sillón, a cuya espalda hay una gran pizarra y la pantalla de proyección. Disponemos de ordenador con conexión a internet y proyector, todo perfectamente conectado e integrado en el espacio. La capacidad de ambas aulas es de 162 puestos (ninguno de ellos adaptado para personas con movilidad reducida) con lo que, teniendo en cuenta la asistencia antes referida, la proporción de puestos es tres libres por cada uno ocupado, lo que genera una gran dispersión en la, libre y voluntaria, colocación de los estudiantes.

La materia impartida, si la consideramos en grandes bloques, serían tres, a saber: Teoría de la Probabilidad y 
Variables Aleatorias, Teoría de Muestras e Inferencia Estadistica (Estimación y Contrastes de Hipótesis) (Cano, 2018). Es una de las asignaturas con mayor tasa de fracaso en el Grado (más del 40\% de los matriculados son repetidores), a mi entender, y sin entrar en más detalles, la razón que lo explica es la abstracción de sus contenidos y la dificultad para que lo relacionen con la realidad.

\section{Diseño previo del Ciclo de Mejora Docente}

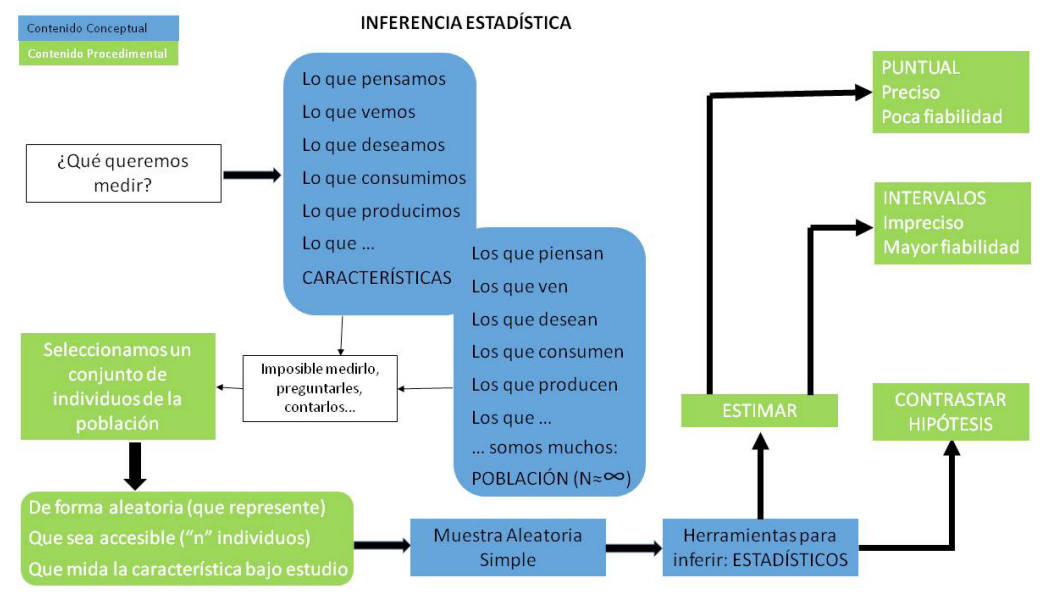

Figura 1. Mapa de contenido para el proceso de Inferencia Estadística.

Partiremos de características que son objeto de nuestro interés, para que podamos trabajarlas con los contenidos de la asignatura hemos de poder definirlas a través de variables aleatorias y serán características que poseen individuos que forman una población, cuando el tamaño de ésta es lo suficientemente grande como para que sea imposible al investigador acceder a todos ellos, seleccionaremos un subconjunto, al que llamaremos muestra, cuidando que sea representativo de toda la población (se lo encargaremos al azar, haciendo la selección de forma aleatoria). Observaremos en los individuos que forman la muestra la característica bajo estudio y le aplicaremos los procesos de Inferencia Estadística. 


\section{Modelo metodológico}

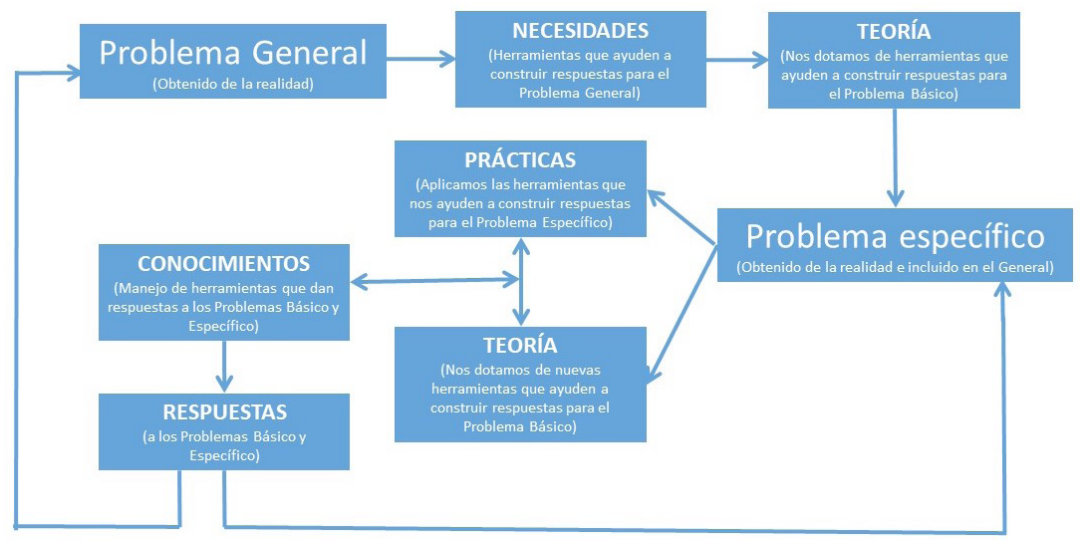

Figura 2. Modelo metodológico.

El ciclo, como describiré con más detalle en el diario de sesiones, arranca con el planteamiento de un problema de actualidad, en nuestro caso el que se ha dado en conocer como La Turistificación, nos propondremos cuantificar algunas de las características que lo definen, y esta propuesta nos llevará al planteamiento del problema específico ¿Perciben los ciudadanos de Sevilla el turismo de masas como una molestia? El desarrollo del ciclo de mejora se diseña como un proceso real encaminado a dar respuesta a dicha pregunta con números reales (Finkel 2008).

El proceso nos demandará herramientas de las que nos dotaremos en clases de teoría y requerirá un trabajo de campo que realizarán los estudiantes fuera del aula. Con el resultado obtenido aplicaremos, en sesiones prácticas, las herramientas de las que nos hemos dotado en teoría.

Jornadas de Formación e Innovación Docente del Profesorado | № 1 (2018)
Esta obra se distribuye con la licencia Creative Commons 


\title{
Secuencia de actividades programadas
}

\author{
Primera Clase
}

\begin{tabular}{|c|c|c|}
\hline Núm. Act.: 01 & Teoría & Duración: 15’ \\
\hline \multicolumn{3}{|c|}{$\begin{array}{l}\text { La caja de herramientas: En las clases precedentes, tras plantear el "Pro- } \\
\text { blema General" (Describir tu barrio con gafas de economista), tuvimos } \\
\text { clases teóricas en formato clase magistral en las que les conté las herra- } \\
\text { mientas básicas con las que construiremos la inferencia (Teoría de la pro- } \\
\text { babilidad, variables aleatorias y modelos probabilísticos) en sustitución de } \\
\text { las herramientas descriptivas. } \\
\text { Los estudiantes intervendrán para hacer el inventario de las herramientas } \\
\text { que disponemos y la utilidad de las mismas. }\end{array}$} \\
\hline \multicolumn{3}{|c|}{ Recursos: Intervenciones voluntarias. } \\
\hline
\end{tabular}

\begin{tabular}{|c|c|c|}
\hline Núm. Act.: 02 & Problema General. & Duración: 15' \\
\hline \multicolumn{3}{|c|}{$\begin{array}{l}\text { Visionado de una noticia de los informativos de TVE1 en el que se pre- } \\
\text { senta el problema de "la turistificación" de las grandes ciudades (entre las } \\
\text { que se encuentra Sevilla. } \\
\text { A continuación les planteo cómo acotamos y medimos el fenómeno que } \\
\text { nos han descrito en el documental. }\end{array}$} \\
\hline \multicolumn{3}{|c|}{$\begin{array}{l}\text { Recursos: } \\
\text { http://www.rtve.es/alacarta/videos/telediario/turistificacion-cuando-turis- } \\
\frac{\text { tas-expulsan-vecinos/4806110/ }}{\text { Intervenciones públicas }}\end{array}$} \\
\hline
\end{tabular}

Jornadas de Formación e Innovación Docente del Profesorado I № 1 (2018) Esta obra se distribuye con la licencia Creative Commons Reconocimiento-NoComercial-SinObraDerivada Internacional (CC BY-NC-ND 4.0.) 
Planteo una sucesión de preguntas:

1. ¿Qué cuestiones aparecen en el documental que sean susceptibles de ser medidas con nuestras herramientas?

2. ¿Qué herramientas de las que tenemos le aplicamos?

3. ¿Cómo lo medimos?

Recursos: Taller conceptual, trabajo en grupos de 4.

\section{Segunda Clase}

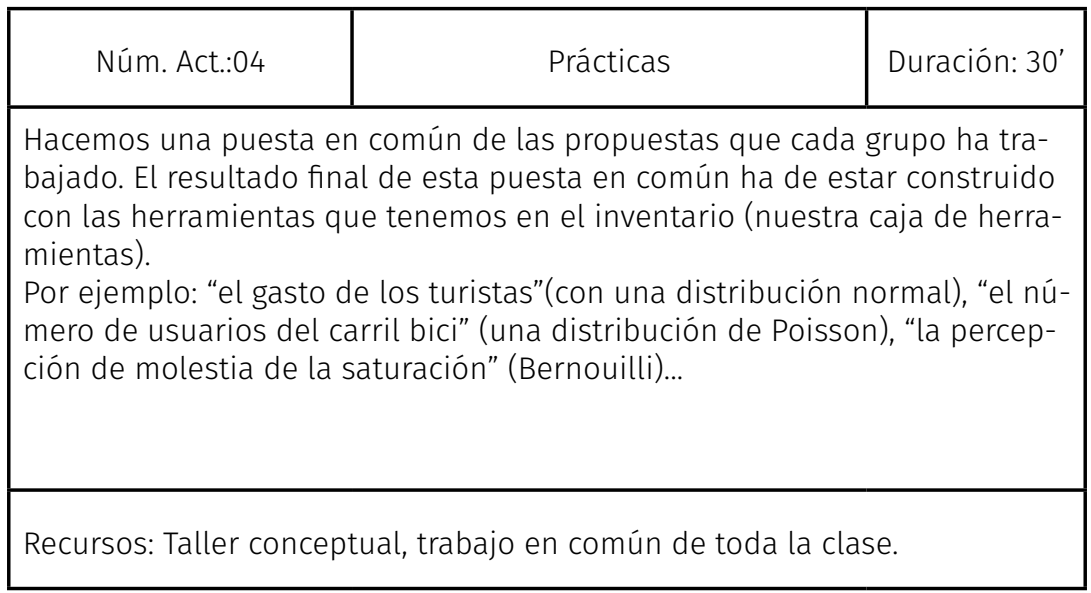

Núm. Act.: 05

Problema Específico

Duración: 15'

De todas las características propuestas seleccionamos las dos más "señaladas" por los estudiantes, que, como no puede ser de otra forma, coinciden con las que más acentúan las publicaciones y reportajes sobre el fenómeno de a turistificación, a saber:

- Los precios de los alquileres

- Las molestias que causa el turismo de masas a los habitantes

Recurso: Trabajo colectivo, dirigido por el profesor con intervenciones de los estudiantes 
Núm. Act.: 06

Problema Específico

Duración: 30'

1. Los estudiantes han de elaborar una propuesta, totalmente especificada, para contar las dos características seleccionadas.

2. Definir las variables que la cuantificarán.

3. Argumentar la distribución que seguirán las variables definidas.

4. Especificar los parámetros de dichas variables.

Recursos: Taller conceptual, trabajo en grupos de 4.

\section{Tercera Clase}

Núm. Act.: 07

Problema Específico

Una vez acordadas las variables a medir (Precios de los alquileres, tanto turísticos como de viviendas y percepción del turismo como molestia)

Les propongo que elaboren el camino a seguir para dar valores a los parámetros desconocidos de las distribuciones de dichas variables (Normal en el caso de los precios, binaria con distribución de Bernouilli en el caso de la percepción)

Recursos: Trabajo fuera del aula y Enseñanza Virtual

Núm. Act.: 08

Teoría

Duración: $30^{\prime}$

Con las propuestas elaboradas por los alumnos construimos las distribuciones de las características acordadas:

- Precio por mes del alquiler de alojamientos turísticos. $€ / \mathrm{m}^{2}$

- Precio por mes del alquiler de alojamientos turísticos. $€ / \mathrm{m}^{2}$

- Variable binaria que toma el valor 1 si el ciudadano declara que el turismo de masas le genera alguna molestia y 0 en cualquier otro caso

Recursos: Taller conceptual, trabajo en común de toda la clase. 
Cuarta Clase

\begin{tabular}{|l|c|c|}
\hline \multicolumn{1}{|c|}{ Núm. Act: 09} & Problema Específico & Duración 60' \\
\hline Unimos los dos grupos en la misma aula para agrupar todas las encuestas, \\
800. Lo que nos permite disponer de un elevado número de datos. \\
Iniciamos el proceso de inferencia que nos lleva a la estimación final del \\
número de Sevillanos a los que el turismo de masas le genera algún tipo de \\
molestias, siendo el valor estimado del 32\%. \\
Recursos: Trabajo de campo y puesta en común en el aula \\
\hline
\end{tabular}

\begin{tabular}{|l|l|l|}
\hline Núm. Act.: 10 & Teoría & Duración: 60' \\
\hline $\begin{array}{l}\text { Tal y como empezamos el ciclo, hacemos un repaso para ordenar el camino } \\
\text { que hemos seguido y rehacer el inventario de herramientas de las que dis- } \\
\text { ponemos. Ahora con una aplicación a un caso real totalmente terminada. }\end{array}$ \\
\hline Recursos: Taller conceptual \\
\hline
\end{tabular}

\section{Cuestionario}

Para la evaluación del ciclo de mejora pasé el mismo cuestionario al inicio y a la finalización del mismo:

En el seno de la Unión europea se está debatiendo la conveniencia de no volver a cambiar la hora en otoño y primavera. El gobierno de España quiere saber el posicionamiento de la ciudadanía al respecto y nos hace el encargo de averiguarlo.

1. ¿Qué propone para responder al gobierno?

2. ¿Cómo llevarías a cabo el plan propuesto en la pregunta anterior? 
3. ¿Cómo articularías la conclusión final de tu propuesta en forma numérica?

\section{Aplicación del CMD}

\section{Clase 1.}

El ciclo de mejora lo arrancamos elaborando un inventario de las herramientas disponibles, básicamente variables aleatorias y sus distribuciones, intentando buscar la utilidad que éstas tienen para poner número a las realidades que nos rodean. Lo que nos llevó a asomarnos a una realidad cercana, les propuse, con la ayuda de un blog y un video, la turistificación, para denominar los problemas que generan en ciudades como Sevilla el turismo de masas.

Les propuse hacer un listado de aquellas características que describieran el turismo en Sevilla y que fuesen susceptibles de ser trabajadas con las herramientas de que disponíamos en el inventario previo, por ejemplo: el gasto de los turistas (con una distribución normal), el número de usuarios del carril bici (una distribución de Poisson), la percepción de molestia de la saturación (Bernouilli)..., descartando aquellas propuestas propias de la Estadística descriptiva, como por ejemplo el número de pernoctaciones en alojamientos hoteleros.

Los estudiantes trabajaron sus propuestas en grupos de 4, pasado un tiempo prudencial hicimos una puesta en común de la que obtuvimos una larga lista de características con sus argumentos y propuestas de distribución, algunas más acertadas y otras un tanto descabelladas, pero la idea era llegar al final a dos propuestas para trabajarlas en el proceso de inferencia.

\section{Clase 2}

La segunda clase la abro, tras haberme llevado a casa la tarea de trabajar las propuestas y elaborar un plan de 
trabajo, con las dos características más destacadas por los estudiantes, en la medida en la que estaban influenciado por el documental y la lectura recomendadas, la propuesta de características fueron los precios de los alquileres y las molestias causadas por el turismo de masas.

Iniciamos un trabajo en grupo para dar forma de variable aleatoria a las características propuestas. La clase se había convertido en un grupo de expertos a los que nos encargaron poner números a esas características (Bain 2007), a modo de ejemplo, cuando se afirma que el turismo de masas genera molestias a los ciudadanos residentes, nuestro papel es cuantificarlo, en este sentido nos encargaremos de poner número a la proporción de residentes en Sevilla a los que el turismo de masas le supone alguna molestia. O cuantificar la influencia del precio de los alquileres turísticos en los de los alquileres residenciales.

El trabajo se encaminó a dar forma a las variables que hemos de utilizar y los parámetros que definen su distribución que, a la postre habremos de estimar.

Una vez definidas las variables y los parámetros:

- $x$ : Normal $\left(\mu_{x^{\prime}}, \sigma_{x}^{2}\right)$ Precio por mes del alquiler de alojamientos turísticos. $€ / \mathrm{m}^{2}$. Donde $\mu_{x}$ es el precio medio de los alquileres turísticos y $\sigma_{x}^{2}$ la varianza de los mismos

- Y: Normal $\left(\mu_{y^{\prime}} \sigma^{2}{ }_{y}\right)$ Precio por mes del alquiler de alojamientos turísticos. $€ / \mathrm{m}^{2}$. Donde $\mu_{y}$ es el precio medio de los alquileres residenciales y $\sigma_{y}^{2}$ su varianza.

- Variable binaria que toma el valor 1 si el ciudadano declara que el turismo de masas le genera alguna molestia y 0 en cualquier otro caso $X$ : Bernouilli $(p)$, donde $p$ sería la proporción de residentes en Sevilla que declara que el turismo de masas le genera alguna molestia.

Todos los parámetros son desconocidos y es nuestra tarea estimarlos y devolver el encargo numérico. Para lo que nos dotamos de las herramientas que la Inferencia 
Estadística pone a nuestra disposición para la estimación paramétrica: Muestra aleatoria Simple, Estadísticos y Estimadores.

\section{Clase 3.}

De las variables seleccionadas, nos quedamos con aquella que supone un camino más fácil para aplicar a la realidad, dadas las limitaciones de las que disponemos.

La variable a trabajar e la que hemos modelizado con una distribución de Bernouilli, de manera que elaboramos una encuesta y se puso a disposición de todos los estudiantes en la plataforma de enseñanza virtual. Antes de esta clase, ellos pasaron la encuesta a 10 personas residentes en Sevilla, intentando simular un procedimiento aleatorio, de manera que nos llevaría a considerar las respuestas al cuestionario como una muestra aleatoria simple. La clase consistió en la explotación de las muestras obtenidas y he de confesar que ha sido una de las mejores clases a la que he asistido en mi carrera profesional, el propio desarrollo de la misma nos fue mostrando el funcionamiento de la muestra, lo que les permitió ver, con el fruto de su propio trabajo, el funcionamiento de los estadísticos definidos a partir de la muestra, la variabilidad de los mismos... en definitiva, entender lo que estábamos haciendo.

Curiosamente, en los dos grupos llegamos a resultados muy similares, en el grupo 1 la estimación de p fue de 0,3274 y en el grupo 3 de 0,3193.

\section{Clase 4.}

La última clase del ciclo la hicimos de forma conjunta con los estudiantes de ambos grupos, para agrupar todas las encuestas, lo que le daba potencia al proceso de estimación, nos permitió disponer de alrededor de 800 encuestas, cifra nada despreciable para un proceso inferencial. 
Cerramos el proceso de estimación de forma más que satisfactoria y dedicamos la última hora del ciclo a recapitular el inventario de herramientas de la que disponíamos ahora.

\section{Evaluación del CMD}

Para contrastar la evolución de la Escalera de Aprendizaje en este ciclo (Porlan, 2017), el cuestionario antes reseñado pretendía ser un continuo en un proceso inferencial, es decir, si las respuestas están bien articuladas, deberían describir un proceso de inferencia estadística de manera que la respuesta a la primera pregunta plantearía la variable, la respuesta a la segunda describiría el proceso a seguir y la tercera culminaría con la estimación. El que sea una sucesión de respuestas articuladas, en vez de compartimentos estancos, ha tenido como consecuencia que las respuestas de los estudiantes se han mezclado, lo que me ha llevado a considerarlas de forma conjunta, en este sentido, he construido una escalera por encuesta, teniendo como resultado una única escalera inicial y otra final.

Las encuestas iniciales se hicieron el viernes 26 de octubre, contestaron 69 alumnos, el ciclo de mejora comenzó en la clase siguiente, martes 30 de octubre, ocupando un total de 8 horas, hasta el martes 13 de noviembre. La encuesta final se realizó el viernes 16 de noviembre y fue respondida por 92 alumnos, de los que solo 57 contestaron a la inicial.

Como ya he señalado anteriormente, al ser una sucesión de preguntas que plantean un proceso continuo, las respuestas se confunden, por lo que he considerado el cuestionario como una única pregunta, agrupando tres grandes grupos y ocho categorías:

A. Respuestas que no están dentro de la categoría de herramientas estadísticas. 
1. Opinión. Aquellos que responden con su opinión personal o acudiendo a la opinión de expertos sin citar herramientas estadísticas.

2. Referéndum. Los que proponen hacer una consulta a todos los ciudadanos, bien mediante referéndum, bien mediante uso de medios de comunicación o redes sociales.

3. Estudio. Los que proponen encargar estudios encaminados a determinar los costes o beneficios del cambio de hora.

B. Respuestas que tienen en cuenta herramientas estadísticas, pero no entran en el campo de la Teoría de la Probabilidad y de Variable Aleatoria.

4. Encuesta. Respuestas centradas en hacer una encuesta sin entrar en su representatividad ni en su distribución de probabilidad.

5. Muestra. Propuesta de selección de una parte de la población, normalmente para hacer una encuesta, pero sin especificar distribución alguna.

6. Variable. Propuestas que especifican alguna distribución de probabilidad, pero no entran en inferencia.

C. Respuestas que tienen en cuenta herramientas estadísticas y entran en el campo de la Teoría de la Probabilidad y de Variable Aleatoria.

7. Proceso de estimación incompleto. Entran en inferencia estadística pero no la especifican o le faltan pasos en el proceso.

8. Proceso de estimación competo. Entran en inferencia estadística con el proceso totalmente (o casi) especificado. 
Cuadro 1. Distribución de las respuestas en categorías:

\begin{tabular}{|c|l|c|c|c|c|}
\hline & & \multicolumn{2}{|c|}{ ENCUESTA INICIAL } & \multicolumn{2}{c|}{ ENCUESTA FINAL } \\
\hline ORDEN & \multicolumn{1}{|c|}{ CATEGORIA } & FRECUENCIA & $\%$ & FRECUENCIA & $\%$ \\
\hline A & OPINIÓN & 13 & 18,84 & 3 & 3,85 \\
\hline B & REFERÉNDUM & 21 & 30,43 & 3 & 3,85 \\
\hline C & ESTUDIO & 7 & 10,14 & 1 & 1,28 \\
\hline D & ENCUESTA & 12 & 17,39 & 7 & 8,97 \\
\hline E & MUESTRA & 11 & 15,94 & 2 & 2,56 \\
\hline F & VARIABLE & 4 & 5,80 & 22 & 28,21 \\
\hline G & $\begin{array}{l}\text { Proceso Estimación } \\
\text { Incompleto }\end{array}$ & 1 & 1,45 & 19 & 24,36 \\
\hline H & $\begin{array}{l}\text { Proceso de Estimación } \\
\text { Completo }\end{array}$ & 0 & 0,00 & 21 & 26,92 \\
\hline & TOTALES & 69 & 100 & 78 & 100 \\
\hline
\end{tabular}

$\%$ Respuestas

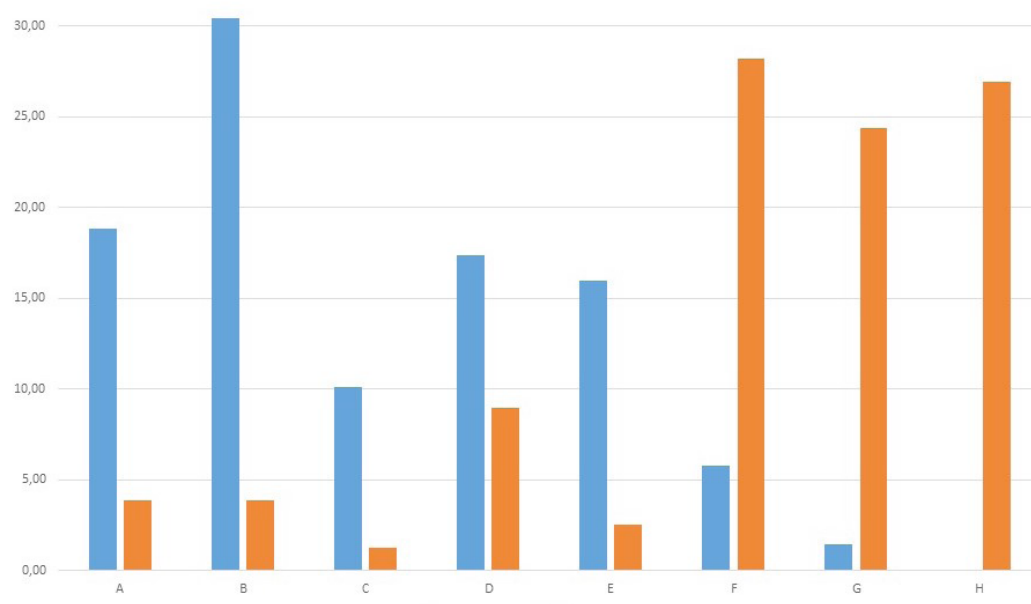

Figura 3. Comparativa de los porcentajes de respuestas en las encuestas iniciales y finales.

Jornadas de Formación e Innovación Docente del Profesorado I № 1 (2018) (c) (i) E $\odot$ Esta obra se distribuye con la licencia Creative Commons 
Porcentaje de respuestas ENCUESTA INICIAL/ENCUESTA FINAL

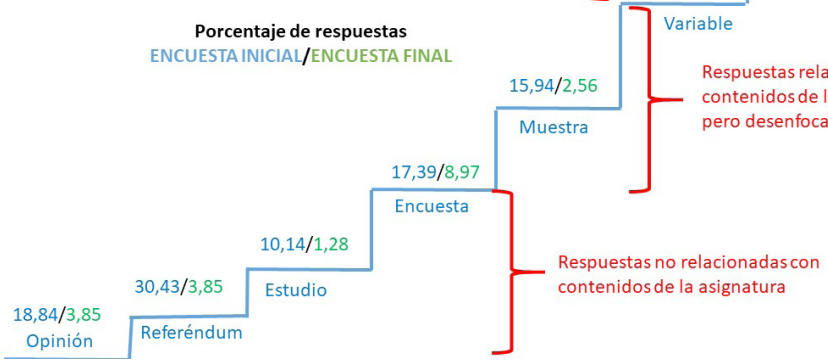

Figura 4. Escalera de aprendizaje.

Cuadro 2. Resultados individuales en las Encuestas Iniciales y Finales.

\begin{tabular}{|c|c|c|c|c|c|c|c|c|c|c|c|c|c|c|c|}
\hline \multirow[b]{2}{*}{$P$} & \multicolumn{2}{|c|}{ Escalón } & \multirow[b]{2}{*}{ Salto } & \multirow[b]{2}{*}{$\mathrm{P}$} & \multicolumn{2}{|c|}{ Escalón } & \multirow[b]{2}{*}{ Salto } & \multirow[b]{2}{*}{$\mathrm{P}$} & \multicolumn{2}{|c|}{ Escalón } & \multirow[b]{2}{*}{ Salto } & \multirow[b]{2}{*}{$\mathrm{P}$} & \multicolumn{2}{|c|}{ Escalón } & \multirow[b]{2}{*}{ Salto } \\
\hline & E.I. & E.F & & & E.I. & E.F & & & E.I. & E.F & & & E.I. & E.F & \\
\hline 1 & 5 & 7 & 2 & 23 & 2 & & & 45 & 2 & 8 & 6 & 67 & 2 & 8 & 6 \\
\hline 2 & 4 & 6 & 2 & 24 & 1 & 4 & 3 & 46 & 6 & 7 & 1 & 68 & 5 & 6 & 1 \\
\hline 3 & 3 & 8 & 5 & 25 & 3 & 6 & 3 & 47 & 3 & 7 & 4 & 69 & 2 & & \\
\hline 4 & 1 & 6 & 5 & 26 & 4 & 8 & 4 & 48 & 3 & 7 & 4 & 70 & & 4 & \\
\hline 5 & 2 & 7 & 5 & 27 & 1 & 1 & 0 & 49 & 4 & 8 & 4 & 71 & & 4 & \\
\hline 6 & 1 & 8 & 7 & 28 & 1 & 8 & 7 & 50 & 3 & 8 & 5 & 72 & & 4 & \\
\hline 7 & 6 & 7 & 1 & 29 & 5 & 7 & 2 & 51 & 5 & 8 & 3 & 73 & & 1 & \\
\hline 8 & 2 & & & 30 & 5 & 8 & 3 & 52 & 5 & 6 & 1 & 74 & & 8 & \\
\hline 9 & 4 & 7 & 3 & 31 & 5 & 8 & 3 & 53 & 2 & 7 & 5 & 75 & & 7 & \\
\hline 10 & 1 & & & 32 & 5 & 8 & 3 & 54 & 2 & & & 76 & & 5 & \\
\hline 11 & 2 & & & 33 & 2 & 5 & 3 & 55 & 7 & & & 77 & & 8 & \\
\hline 12 & 4 & & & 34 & 2 & 6 & 4 & 56 & 4 & 7 & 3 & 78 & & 8 & \\
\hline 13 & 4 & 8 & 4 & 35 & 2 & 7 & 5 & 57 & 1 & 6 & 5 & 79 & & 7 & \\
\hline 14 & 2 & & & 36 & 2 & 6 & 4 & 58 & 2 & 8 & 6 & 80 & & 7 & \\
\hline 15 & 2 & 4 & 2 & 37 & 6 & & & 59 & 4 & 8 & 4 & 81 & & 7 & \\
\hline 16 & 4 & 6 & 2 & 38 & 2 & 7 & 5 & 60 & 1 & 6 & 5 & 82 & & 7 & \\
\hline 17 & 1 & 8 & 7 & 39 & 5 & & & 61 & 6 & 7 & 1 & 83 & & 2 & \\
\hline
\end{tabular}

Jornadas de Formación e Innovación Docente del Profesorado | № 1 (2018) 


\begin{tabular}{llll|llll|l|lll|ll|}
18 & 1 & 1 & 0 & 40 & 3 & 6 & 3 & 62 & 2 & 8 & 6 & 84 & 2 \\
19 & 4 & 6 & 2 & 41 & 5 & 7 & 2 & 63 & 4 & 8 & 4 & 85 & 6 \\
20 & 1 & 4 & 3 & 42 & 2 & 4 & 2 & 64 & 1 & 3 & 2 & 86 & 6 \\
21 & 1 & 6 & 5 & 43 & 3 & 6 & 3 & 65 & 2 & 6 & 4 & 87 & 6 \\
22 & 2 & & 44 & 5 & 6 & 1 & 66 & 2 & 2 & 0 & 88 & 6 \\
89 & 6 \\
90 & 6
\end{tabular}
P. Participante

E.F.: Posición en la Encuesta Final

\section{Evaluación del diseño del ciclo de mejora}

Si establecemos una correspondencia ordinal para las categorías establecidas, asignando el valor 1 a la más alejada de la respuesta correcta (categoría A) y 8 (Categoría H) a la a más adecuada, podemos hacer un análisis ordinal de la distribución de respuesta, bajo el supuesto de que los saltos de escalón a escalón son lineales.

Las respuestas en la Encuesta Inicial se situarían, por término medio en el escalón 3, es decir no sobrepasaría el grupo que no considera las herramientas de estadística. Siendo el valor más frecuente el 2, el que propone hacer una consulta (Referéndum) entre los ciudadanos, no encontrando ninguna respuesta que alcance el valor máximo y solo 5 en el grupo de herramientas ligadas a Teoría de Probabilidad y Variable Aleatoria, herramientas que ya se han trabajado en el primer Ciclo de Mejora, sin embargo, aun habiéndose trabajado no se habían relacionado con procesos de inferencia. Entiendo que esta es la razón por la que los estudiantes, de forma masiva, no eran capaces de relacionar herramientas y soluciones al problema planteado

En este ciclo de mejora, partiendo de un problema específico similar al que encabezaba la encuesta, hemos recorrido un camino real en el que relacionamos herramientas (Teoría de la Probabilidad y Variables Aleatorias) e inferencia de características poblacionales, lo que les ha 
permitido dar un salto importante en la escalera de aprendizaje, como podemos comprobar en los valores obtenidos.

Las respuestas en la Encuesta final se sitúan en el escalón 6,4 por término medio, lo que supone un salto de 3,4. En comparación con la Encuesta Inicial, la mayoría de las respuestas (más del 80\%) se sitúa en el grupo más alto, el que usa las herramientas trabajadas en clase.

A la vista de los resultados comparativos, podemos concluir, sin lugar a dudas, que el segundo ciclo de mejora ha tenido un resultado que podemos calificar de muy bueno.

\section{Conclusiones}

Este ciclo de mejora es el primer intento por vencer los vicios adquiridos durante años de docencia siguiendo una metodología tradicional y cuyos resultados, como ya destaqué en la descripción del contexto, podemos calificar de desastrosos a la vista del alto número de repetidores $y$, sobre todo, de la dificultad que tienen los estudiantes para aprender los contenidos de una materia como la que nos ocupa, amén de las dificultades para comunicarnos en la transmisión de estos conocimientos. La metodología puesta en práctica en este CMD, en la medida que los alumnos han manejado la herramienta real y han construido todo un proceso en el que iban necesitando herramientas para seguir adelante, se les abría las puertas del entendimiento, con lo que conceptos que antes parecían inaccesibles a la comprensión han llegado a sus mentes para quedarse, o al menos para ser almacenados en la carpeta de herramientas para aplicar, ahora disponen de herramientas útiles para explicar realidades, antes tenían un cajón de conceptos amontonados esperando a ser preguntados en un examen para salir de sus mentes sin retorno.

El ciclo de mejora que hemos desarrollado tiene muchas puertas abiertas para seguir mejorando, en la metodología, en las actividades, en la evaluación..., pero lejos 
de anclarse en un bucle de repetición, como ha venido ocurriendo en la tradicional forma de dar clases, nos abre un camino ilusionante que nos llama a emprender nuevas vías cada curso.

\section{Bibliografía}

Bain, K. (2007). Lo que hacen los mejores profesores de universidad. Valencia: Universitat de Valencia.

Cano, A. (2018). Estadística Avanzada. 3 ${ }^{\mathrm{a}}$ Ed. Red de Impresión.

Finkel, D. (2008). Dar clase con la boca cerrada. Valencia: Universitat de Valencia.

Porlán, R. (2017). Enseñanza universitaria. Cómo mejorarla. Ediciones Morata, S. L.

Jornadas de Formación e Innovación Docente del Profesorado | № 1 (2018) Esta obra se distribuye con la licencia Creative Commons 